\title{
Refined identification of neutralization-resistant CRF02_AG viruses and their sensitivity to anti- MPER neutralizing antibodies
}

\author{
RA Jacob ${ }^{*}$, F Abrahams ${ }^{1}$, M Tongo ${ }^{2}$, M Schomaker ${ }^{3}$, P Roux ${ }^{4}$, E Mpoudi Ngole ${ }^{5}$, WA Burgers ${ }^{2}$, JR Dorfman ${ }^{1}$
}

From AIDS Vaccine 2012

Boston, MA, USA. 9-12 September 2012

\section{Background}

The first antibody-inducing HIV-1 vaccines are unlikely to protect against all HIV-1 isolates. There is thus a danger that a vaccine will select for HIV-1 viruses that are highly resistant to antibody-mediated neutralization. We sought to identify and characterize such viruses.

\section{Methods}

A diverse panel of 24 HIV-1 pseudoviruses was tested for neutralization resistance using two sets of samples from ARV-naive HIV-1-infected individuals selected for good neutralizers: sera from South Africa donors $(\mathrm{n}=68$, infected $>1$ year, subtype C predominant area) and CRF02_AGinfected plasma samples from Cameroon donors $(\mathrm{n}=12$, good neutralizers selected from 22 samples).

\section{Results}

Sensitivity to South Africa sera by subtype was $\mathrm{C}>\mathrm{B} \approx \mathrm{CRF} 02 \_\mathrm{AG}>\mathrm{A}$. Importantly, and in contrast to previous reports, CRF02_AG plasma neutralized CRF02_AG viruses better than other panel viruses ("within-subtype neutralization"). This included three (257-31, 251-18 and 33-7) of five CRF02_AG viruses previously designated as tier 3 (most resistant). This within-subtype neutralization testing showed that the other two tier 3 CRF02_AG panel viruses, 253-11 and 278-50 were highly resistant. Most CRF02_AG viruses, including 253-11 and 278-50 were sensitive to two membrane proximal external region (MPER)-specific monoclonal antibodies and soluble CD4 (sCD4), suggesting targets for neutralization of even these highly resistant viruses. This information may help design a global HIV-1 vaccine. We also propose testing viruses with within-subtype samples selected for good neutralizers in order to evaluate their neutralization resistance.

\section{Conclusion}

Some but not all CRF02_AG viruses are sensitive to neutralisation by CRF02_AG-derived plasma, even though most are previously reported as highly resistant (Tier 3). Further work is necessary to properly characterize such Tier 3 viruses. If research focus is not placed on such resistant viruses, a future partially effective HIV-1 vaccine may select for them.

\section{Author details}

${ }^{1}$ International Centre for Genetic Engineering and Biotechnology, Cape Town, South Africa. ${ }^{2}$ Division of Medical Virology, University of Cape Town, Cape Town, South Africa. ${ }^{3}$ Centre for Infectious Disease Epidemiology \& Research, UCT, Cape Town, South Africa. ${ }^{4}$ School of Child and Adolescent Health, University of Cape Town, Cape Town, South Africa. ${ }^{5}$ Centre de Recherche sur les Maladies Émergentes et Réémergentes, Yaoundé, Cameroon.

Published: 13 September 2012

doi:10.1186/1742-4690-9-S2-P77

Cite this article as: Jacob et al: Refined identification of neutralizationresistant CRF02_AG viruses and their sensitivity to anti-MPER neutralizing antibodies. Retrovirology 2012 9(Suppl 2):P77. 